\title{
Cloud Computing - Von Enterprise Systems zu Business Networks
}

\author{
Hans-Peter Fröschle • Stefan Meinhardt
}

Eingegangen: 20. Oktober 2014 / Angenommen: 23. Oktober 2014

(C) Springer Fachmedien Wiesbaden 2014

Cloud Computing als ein im Wesentlichen auf der Virtualisierung von IT-Infrastrukturkomponenten basierenden IT-Betriebsmodell bietet für die IT-Industrie neue Möglichkeiten, IT-Ressourcen nicht mehr lokal (On Premise) selbst zu betreiben, sondern als Services über das Internet oder in Intranets bedarfsorientiert zu nutzen. Abgerechnet wird nach dem tatsächlichen Ressourcenverbrauch. Gesteigerte Flexibilität sowie nahezu unbegrenzte Skalierbarkeit und im Endeffekt niedrigere Kosten sind erwartete Effekte. Ein derartiger Paradigmenwechsel im IT-Betrieb

- ist verbunden mit tiefgreifenden Auswirkungen auf die IT-Industrie und die etablierten Geschäftsmodelle,

- erfordert auf Nutzerseite weitreichende Entscheidungen, welche Applikationen unter Wettbewerbsgesichtspunkten ausgelagert bzw. weiterhin selbst betrieben werden (USP versus Skaleneffekte) und

- ist immer vor dem Hintergrund von Datenschutz und -sicherheit sowie der Verfügbarkeit der Ressourcen zu bewerten.

Die Auswirkungen auf die IT-Industrie beschreiben Sven Denecken und Bert Schulze am Beispiel der SAP AG. Software-Anbieter werden zunehmend zu Serviceprovidern und müssen ihr komplettes Leistungsspektrum viel stärker auf die Kunden ausrichten als in der Vergangenheit. Kundenorientierung wird damit vom Schlagwort

\footnotetext{
H.-P. Fröschle $(\bowtie)$

i.t-consult $\mathrm{GmbH}$,

Pischekstrasse 68,

70184 Stuttgart, Deutschland

E-Mail: hpf@i-t-consult.de

S. Meinhardt

SAP AG,

Neurottstr. 16,

69190 Walldorf, Deutschland

E-Mail: stefan.meinhardt@sap.com
} 
zum zentralen Bestandteil neuer Geschäftsmodelle in der IT-Industrie. Langfristige und nachhaltige Kundenbeziehungen erfordern eine Intensivierung der Zusammenarbeit zwischen den Geschäftspartnern über den gesamten Service-Lifecycle.

Auch auf Anwenderseite sind mit der Nutzung von Cloud Computing gravierende Änderungen für die Fachbereiche und die IT verbunden. Martin Andenmatten arbeitet in seinem Beitrag heraus, wie Aufgaben und Verantwortlichkeiten zwischen einer „Retained IT“ und einem externen Service-Provider neu zu definieren sind, welche Rahmenbedingungen für eine entsprechende Outsourcing-Entscheidung unbedingt zu berücksichtigen sind und welche organisatorischen und vor allem qualifikatorischen Änderungen innerhalb der unternehmensinternen IT erforderlich sind.

Neben Diskussionen um technische, wirtschaftliche und organisatorische Aspekte wird das Thema Cloud Computing derzeit vor allem durch Sicherheits- und rechtliche Fragestellungen geprägt. So verbieten deutsche und europäische Datenschutzgesetze die unverschlüsselte Speicherung von vor allem personenbezogenen Daten in Rechenzentren und auf Servern außerhalb des Europäischen Wirtschaftsraumes. Wie trotzdem eine gesetzeskonforme und den Compliance-Anforderungen der Unternehmen gerecht werdende Lösung unter juristischen und vertraglichen Gesichtspunkten aussehen kann, erläutern Deister und Meyer-Spasche in ihrem Beitrag.

Vor dem Hintergrund der vorliegenden Beiträge wird deutlich, dass cloud-basierte IT-Betriebsmodelle zunehmend realisiert werden (können) und diese Entwicklung tatsächlich einen grundlegenden Paradigmenwechsel für die gesamte IT-Welt bedeuten. 